\section{Efficacy of cyclosporine for treatment induction of canine pemphigus foliaceus}

\section{T. Olivry, C. RivierRe, K. M. MURPhy}

CYCLOSPORINE (ciclosporine, cyclosporin A, CsA) is a cyclic oligopeptide which exhibits potent immunosuppressive properties by its ability to block the transcription of cytokine genes in activated T cells (Matsuda and Koyasu 2000). Because of its suppressive effect on immune cell activation, cyclosporine is being used with increasing frequency for the treatment of autoimmune and allergic skin diseases. Several case reports and small case studies have reported the benefit of cyclosporine for the treatment of human variants of pemphigus, an autoimmune disease directed against interkeratinocyte desmosomal adhesion molecules (Thivolet and others 1985 , Balda and Rosenzweig 1986, Barthelemy and others 1988, Alijotas and others 1990, Bondesson and Hammar 1990, Campolmi and others 1991, Lapidoth and others 1994, Luisi and Stoukides 1994, Mobini and others 1997). In these studies, cyclosporine was effective either as a stand-alone treatment modality, or in combination with low immunosuppressive doses of oral glucocorticoids. Recently, a larger case study disputed the earlier findings of efficacy, as cyclosporine was found to offer no additional advantage over oral glucocorticoid monotherapy (Ioannides and others 2000). Thus, the issue of whether cyclosporine is of value in the treatment of human pemphigus variants remains uncertain.

To date, the standard of care for treatment of canine pemphigus foliaceus (PF), the most common variant of pemphigus in animals, involves immunosuppression with oral glucocorticoids used alone or in combination with cytotoxic drugs such as azathioprine (Olivry and Chan 2001). Unfortunately, the prolonged use of these medications is often followed by potentially life-threatening adverse drug effects.

This short communication describes a pilot study to investigate whether cyclosporine monotherapy would be effective for the induction of treatment in dogs with PF.

Five dogs diagnosed with PF between June 2000 and February 2001 at the North Carolina State University Veterinary Teaching Hospital, USA, were selected for this clinical trial. In each case, the diagnosis of PF was based on suggestive clinical signs, diagnostic histopathology, and the presence of skin-fixed and circulating anti-keratinocyte autoantibodies in direct and indirect immunofluorescence assays, respectively (Olivry and Chan 2001). In addition, other pustular skin diseases, such as superficial pyoderma and pustular dermatophytosis, were ruled out using standard diagnostic and/or therapeutic methods.

To document the severity of clinical signs of PF at each $152,53-54$

T. Olivry, DrVet, PhI), DipACVD, DipECVD, C. Rivierre, DrVet, MspVM, DipE(CVI), DipACVI),

K. M. Murphy, 1)VM, DipACVD,

Department of Clinical Sciences, College of Veterinary Medicine, North Carolina State University, Raleigh, NC 27606, USA tem consisted of the evaluation of the severity ( 0 none, 1 mild 2 moderate and 3 severe) of the three cardinal lesions of $\mathrm{PF}$ (pustules, erosions and crusts) at 50 different areas covering the entire body surface. Even though the maximum PFFESI pedal predominant distribution of $\mathrm{pF}$ skin lesions usually results in a much lower score. Indeed, preliminary scoring of pretreatment skin lesions in 16 dogs with PF yielded PEFESI values ranging from 8 to 128 (mean 49, median 41) (T. Olivry, unpublished data). The primary outcome measure of this pilot trial was the reduction from the baseline of the PEFESI lesional score.

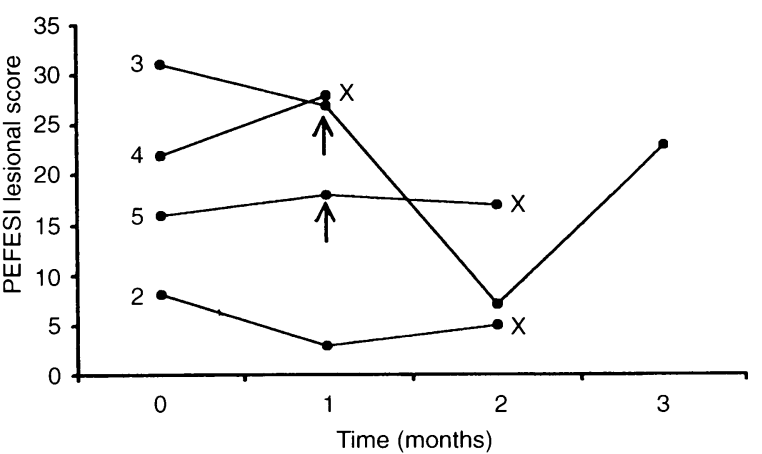

FIG 1: Pemphigus foliaceus extent and severity index (PEFESI) scores of dogs 2 to 5 during the trial. The arrows indicate a doubling of the dose of cyclosporine to $10 \mathrm{mg} / \mathrm{kg} / \mathrm{day}$ : $X$ indicates withdrawal of the dog from the study

The therapeutic intervention consisted of the sole administration of $5 \mathrm{mg} / \mathrm{kg}$ oral cyclosporine capsules (NEORAL; Novartis-Pharma) once a day. At each monthly re-evaluation visit, if there was a reduction in the lesional score of more than 50 per cent, the medication was continued at the same dose for another month. If there was a lack of appreciable improvement, the dose of cyclosporine was doubled to 10 $\mathrm{mg} / \mathrm{kg}$ once a day. The owners could elect to remove their pet from the study whenever they felt that the clinical signs worsened in spite of the treatment. The trial lasted three months.

A description of the five dogs entered in the study is given in Table 1, and the lesional scores of dogs 2 to 5 during the trial are shown in Fig 1.

The owner of dog 1 thought the treatment was ineffective after two weeks, and therefore doubled the dosage of cyclosporine. However, after another two weeks the owner considered that the clinical signs had not improved, and requested the prescription of glucocorticoids, without further evaluation in the study.

The PEFESI values of $\operatorname{dog} 2$, although low, were reduced by 63 per cent after receiving cyclosporine for one month; the medicine was continued at the same dose. Because of a deterioration in clinical signs, the dog was removed from the study after two months of cyclosporine monotherapy.

The lesional scores of dog 3 had only decreased by 13 per cent after one month and so the dose of cyclosporine was doubled. In the following month, the PEFEs had dropped to 77 per cent from baseline scores and the drug was continued at the same dose. By the end of the trial, the PEFESI had increased and its value was reduced by only 26 per cent from the baseline.

The PEFFSI values of $\operatorname{dog} 4$ increased by 27 per cent during the first month of cyclosporine monotherapy, and the owner chose to withdraw the animal from the trial.

The lesional scores of dog 5 increased by 13 per cent after one month of treatment, so the dose of cyclosporine was doubled. After another month, the PEFESI had only decreased by one point and was still 6 per cent above the baseline value. This patient also was withdrawn from the study at the owner's request. score theoretically achievable is $450(3 \times 3 \times 50)$, the facial-

\begin{tabular}{|c|c|c|c|}
\hline Dog & Breed & Sex & Age at start of trial (years) \\
\hline 1 & Basset hound cross & FN & 7 \\
\hline 2 & Crossbreed & MN & 3 \\
\hline 3 & Bulldog & $M$ & 4 \\
\hline 4 & Chow cross & $M N$ & 3 \\
\hline 5 & Labrador cross & FN & 2 \\
\hline
\end{tabular}

FN Neutered female, MN Neutered male, M Male 
The adverse drug effects throughout the trial were minimal, with intermittent diarrhoea only reported for dog 1 .

In this short-duration pilot study, the induction of treatment with 5 to $10 \mathrm{mg} / \mathrm{kg}$ of cyclosporine once daily was unable to lead to complete remission of PF in any of the dogs. Moreover, four of the five dogs had to be withdrawn from the study because of exacerbation of lesional scores. Because of this lack of noticeable efficacy, administration of cyclosporine at the proposed dose cannot be recommended for treatment of $\mathrm{PF}$ in dogs. However, it is possible that higher doses of cyclosporine could be more effective than those used in this study. Future trials should investigate whether cyclosporine would be a useful adjuvant medication, helping to lower the dose of glucocorticoids when both drugs are administered concurrently during the induction phase of immune suppression.

\section{ACKNOWLEDGEMENTS}

The authors are grateful to Mr Jean Steffan (Novartis Animal Health, Basel, Switzerland) for providing the oral cyclosporine used to treat the dogs in this study.

\section{References}

ALIJOTAS, J., PEDRAGOSA, R., BOSCH, J. \& VILARDELL, M. (1990) Prolonged remission after cyclosporine therapy in pemphigus vulgaris. Journal of the American Academy of Dermatology 23, 701-703

BALDA, B. R. \& ROSENZWEIG, D. (1986) Cyclosporin A in the treatment of pemphigus foliaceus and pemphigus erythematosus. Hautarzt 37, 454-457 BARTHELEMY, H., FRAPPAZ, Z., CAMBAZARD, F., MAUDUIT, G., ROUCHOUSE, B., KANITAKIS, J., SOUTEYRAND, P., CLAUDY, A. L. \& THIVOLET, J. (1988) Treatment of nine cases of pemphigus vulgaris with cyclosporine. Journal of the American Academy of Dermatology 18, 1262-1266 BONDESSON, L. \& HAMMAR, H. (1990) Treatment of pemphigus vulgaris with cyclosporine. Dermatologica 181, 308-310

CAMPOLMI, P., BONAN, P., LOTTI, T., PALLESCHI, G. M., FABBRI, P. \& PANCONESI, E. (1991) The role of cyclosporine A in the treatment of pemphigus erythematosus. International Journal of Dermatology 30, 890-892

IOANNIDES, D., CHRYSOMALLIS, F. \& BYSTRYN, J.C. (2000) Ineffectiveness of cyclosporine as an adjuvant to corticosteroids in the treatment of pemphigus. Archives of Dermatology 136, 868-872

LAPIDOTH, M., DAVID, M., BEN-AMITAI, D. V. K., LUSTIG, S. \& SANDBANK, M. (1994) The efficacy of combined treatment with prednisone and cyclosporine in patients with pemphigus: a preliminary study. Journal of the American Academy of Dermatology 30, 752-757

LUISI, A. F. \& STOUKIDES, C. A. ( 1994) Cyclosporine for the treatment of pemphigus vulgaris. Annals of Pharmacotherapy 28, 1183-1185

MATSUDA, S. \& KOYASU, S. (2000) Mechanisms of action of cyclosporine. Immunopharmacology 47, 119-125

MOBINI, N., PADILLA, T. \& AHMED, A. R. (1997) Long-term remission in selected patients with pemphigus vulgaris treated with cyclosporine. Journal of the American Academy of Dermatology 36, 264-266

OLIVRY, T. \& CHAN, L. S. (2001) Spontaneous animal models of autoimmune blistering dermatoses. Clinics in Dermatology 19,750-760

THIVOLET, J., BARTHELEMY, H., RIGOT-MULLER, G. \& BENDELAC, A (1985) Effects of cyclosporin on bullous pemphigoid and pemphigus. Lancet 8424, 334-335

\section{Notices and divisional events}

DIVISIONS of the BVA are entitled to a free notice in The Veterinary Record for each meeting that they organise. Notices should contain the date, time, venue and town of the meeting, details of the subject, any speakers and sponsors and the address and telephone number of the person from whom details can be obtained. They should be addressed to: Kathryn Clark, The Veterinary Record, 7 Mansfield Street, London WIG 9NQ, fax 02076370620.

\section{Mammary botryomycosis and mastectomy in an African elephant (Loxodonta africana)}

\author{
J. M. Sleeman, V. L. Clyde, M. V. Finnegan, \\ E. C. RAMSAY, M. G. SHIRES
}

AFRICAN elephants (Loxodonta africuna) and Asian elephants (Elephas maximus) are susceptible to a number of systemic bacterial infections including anthrax, salmonellosis, tuberculosis, tetanus, pasteurellosis, clostridial infections, colibacillosis, and diphtheria (Schmidt 1986). In addition, elephants are prone to skin and subcutaneous abscess formation due to to the thickness and toughness of their integument (Schmidt 1986). Elephants have paired mammary glands, which are located in the axillary region. There is increasing interest in elephant reproduction (Schmidt 1993) and the normal anatomy of a lactating mammary gland has been described (Welsch and others 1998) but to the authors' knowledge, there are no reports of diseases of the mammary glands of elephants. This short communication describes the clinical presentation and surgical management of a case of mammary botryomycosis in a captive African elephant.

In 1987, a 14-year-old, female African elephant housed at the Knoxville Zoological Gardens, Tennessee, USA, developed an enlargement of the left mammary gland. In 1989, Staphylococcus aureus was cultured from purulent material expressed from the gland and a course of $60 \mathrm{~g}$ of trimethoprim/sulfadiazine (Tribrissen 400; Schering Plough Animal Health) was given orally once a day for 10 days. Due to the lack of response to therapy an excisional biopsy was performed under sedation. Histopathologically, multifocal granulomas with centrally located Splendore-Hoeppli bodies and associated eosinophils, and epithelioid macrophages were identified; however, no aetiological agents were seen. No further treatment was instituted and the mass continued to slowly expand.

In 1992, the mass measured $20 \mathrm{~cm} \times 10 \mathrm{~cm} \times 8 \mathrm{~cm}$ and a repeat biopsy was performed under sedation which revealed fibrous connective tissue with lymphocytic-plasmacytic perivasculitis. At this time it was elected to perform surgical excision of the affected mammary gland. The elephant was fasted for 24 hours and anaesthesia was induced with an intramuscular injection of $6 \mathrm{mg}$ of carfentanil citrate (Wildnil; Wildlife Pharmaceuticals), followed by a further dose of $3 \mathrm{mg}$. The animal was guided into right lateral recumbency using ropes and anaesthesia was maintained using repeated doses of 0.5 to $1.0 \mathrm{mg}$ of carfentanil citrate, which was administered intravenously at approximately 15 minute intervals. The elephant was intubated with a $30 \mathrm{~mm}$ diameter endotracheal tube and oxygen was delivered at 20 litres/minute.

The left mammary area was aseptically prepared and a 25 $\mathrm{cm}$ curvilinear incision was made medial to the right nipple and centered over the enlarged mammary gland. Electrosurgical dissection of the mass from the skin and underlying musculature was performed. Multiple small vessels were cauterised and two larger vessels were ligated using number 2 polyglactin 910 thread (Vicryl; Ethicon). A second curvilinear incision was made lateral to the nipple so that it, the associated tissues and underlying mass could be more readily removed (Fig 1). The space was packed with gauze soaked in povidone-iodine (Betadine; Purdue Frederick
Veterinary Record (2003) 152, 54-55

\section{J. M. Sleeman, VetMB} DiplACZM, MRCVS, Wildlife Center of Virginia, PO Box 1557 Waynesboro, Virginia 22980, USA

V. L. Clyde, DVM, Milwaukee County Zoo 1001 Blue Mound Road Milwaukee, Wisconsin 53226, USA

M. V. Finnegan, DVM, Oregon Zoo, 4001 SW Canyon Road, Portland, Oregon 97221, USA E. C. Ramsay, DVM, DiplACZM,

Department of Comparative Medicine, M. G. Shires, DVM, DiplACVS, College of Veterinary Medicine, University of Tennessee, PO Box 1071, Knoxville, Tennessee 37901-1071, USA 